\title{
Microlocal analysis of fractional wave equations
}

\author{
Günther Hörmann*, Ljubica Oparnicał, Dušan Zorica ${ }^{\ddagger}$
}

February 25, 2018

\begin{abstract}
We determine the wave front sets of solutions to two special cases of the Cauchy problem for the space-time fractional Zener wave equation, one being fractional in space, the other being fractional in time. For the case of the space fractional wave equation, we show that no spatial propagation of singularities occurs. For the time fractional Zener wave equation, we show an analogue of non-characteristic regularity.

Key words: wave front set, space-time fractional wave equation, Cauchy problem, fractional Zener model, fractional strain measure
\end{abstract}

\section{Introduction}

This paper is devoted to the microlocal analysis of the solution to the generalized Cauchy problem for the space-time fractional Zener wave equation

$$
Z u(x, t)=\partial_{t}^{2} u(x, t)-L_{t}^{\alpha} \partial_{x} \mathcal{E}_{x}^{\beta} u(x, t)=u_{0}(x) \otimes \delta^{\prime}(t)+v_{0}(x) \otimes \delta(t)
$$

considered as an equation on all $\mathbb{R}^{2}$ with $\operatorname{supp}(u) \subseteq\left\{(x, t) \in \mathbb{R}^{2} \mid t \geq 0\right\}$ and $u_{0}, v_{0} \in \mathcal{E}^{\prime}(\mathbb{R})$. The generalized Cauchy problem (1) is derived and analyzed in [1], where existence and uniqueness of distributional solutions has been shown. In the present paper we study the wave front set for special cases of (1) when $\alpha=0$, or $\beta=1$.

The operators $L_{t}^{\alpha}$ and $\mathcal{E}_{x}^{\beta}$ are of convolution type, with respect to one variable only, denoted by $*_{t}$ and $*_{x}$, respectively, and act on a distribution $w=w(x, t)$ in the following way

$$
\begin{aligned}
L_{t}^{\alpha} w & =\mathcal{F}_{\tau \rightarrow t}^{-1}\left[\frac{1+b \mathrm{e}^{\mathrm{i} \frac{\alpha \pi}{2}}(\tau-\mathrm{i} 0)^{\alpha}}{1+a \mathrm{e}^{\mathrm{i} \frac{\alpha \pi}{2}}(\tau-\mathrm{i} 0)^{\alpha}}\right] *_{t} w, \quad \alpha \in[0,1), 0<a<b, \\
\mathcal{E}_{x}^{\beta} w & =\mathcal{F}_{\xi \rightarrow x}^{-1}\left[i \sin \left(\frac{\beta \pi}{2}\right) \operatorname{sgn}(\xi)|\xi|^{\beta}\right] *_{x} w, \quad \beta \in(0,1) .
\end{aligned}
$$

Note that $\mathcal{E}_{x}^{1} w=\frac{\partial}{\partial x} w$ and, in case $0<\beta<1$, we have

$$
\mathcal{E}_{x}^{\beta} w=\frac{1}{2 \Gamma(1-\beta)} \frac{1}{|x|^{\beta}} *_{x} \frac{\partial}{\partial x} w .
$$

\footnotetext{
*Faculty of Mathematics, University of Vienna, Oskar-Morgenstern-Platz 1, 1090 Wien, Austria, guenther.hoermann@univie.ac.at

$\dagger^{\dagger}$ Faculty of Education in Sombor, University of Novi Sad, Podgorička 4, 25000 Sombor, Serbia, ljubica.oparnica@gmail.com

¥Mathematical Institute, Serbian Academy of Arts and Sciences, Kneza Mihaila 36, 11000 Belgrade, Serbia, dusan_zorica@mi.sanu.ac.rs and Department of Physics, Faculty of Sciences, University of Novi Sad, Trg D. Obradovića 4, 21000 Novi Sad, Serbia
} 
The operator $L_{t}^{\alpha}$, considered as a convolution operator in one variable, is linear and bounded $L^{p}(\mathbb{R}) \rightarrow L^{p}(\mathbb{R}), 1<p<\infty$, by Hörmander's multiplier theorem (cf. [3, Corollary 8.11] or [5, Theorem 7.9.5]), since $l_{\alpha}$, defined by

$$
l_{\alpha}(\tau)=\frac{1+b \mathrm{e}^{\mathrm{i} \frac{\alpha \pi}{2}}(\tau-\mathrm{i} 0)^{\alpha}}{1+a \mathrm{e}^{\mathrm{i} \frac{\alpha \pi}{2}}(\tau-\mathrm{i} 0)^{\alpha}}
$$

is in $L^{\infty}(\mathbb{R}) \cap C^{1}(\mathbb{R} \backslash\{0\})$ with derivative bounded by a constant times $|\tau|^{-1}$. Note that in [1], the operator $L_{t}^{\alpha}$ is employed in its Laplace transform variant $L_{t}^{\alpha} w=\mathcal{L}_{s \rightarrow t}^{-1}\left[\frac{1+b s^{\alpha}}{1+a s^{\alpha}}\right] *_{t} w$.

The operator $\mathcal{E}_{x}^{\beta}$, acting by convolution in one variable with $h(x):=|x|^{-\beta}$ (apart from a constant), following a differentiation, is a bounded linear operator $W^{1, p}(\mathbb{R}) \rightarrow L^{q}(\mathbb{R}), 1<p<q<\infty$ and $\frac{1}{p}+\beta=\frac{1}{q}+1$, since by the Hardy-Littlewood-Sobolev-inequality [5, Theorem 4.5.3] the map $w \mapsto h * w$ is continuous $L^{p}(\mathbb{R}) \rightarrow L^{q}(\mathbb{R})$ in this setting.

The space-time fractional Zener wave equation

$$
Z u(x, t):=\partial_{t}^{2} u(x, t)-L_{t}^{\alpha} \partial_{x} \mathcal{E}_{x}^{\beta} u(x, t)=0, \quad x \in \mathbb{R}, t>0,
$$

subject to initial conditions

$$
u(x, 0)=u_{0}(x), \frac{\partial}{\partial t} u(x, 0)=v_{0}(x), \quad x \in \mathbb{R},
$$

is derived in [1] from the system of three equations: The equation of motion of a (one-dimensional) deformable body, the constitutive equation and the non-local strain measure. In dimensionless form, the system of equations reads

$$
\begin{aligned}
\frac{\partial}{\partial x} \sigma(x, t) & =\frac{\partial^{2}}{\partial t^{2}} u(x, t), \\
\sigma(x, t)+a_{0} \mathrm{D}_{t}^{\alpha} \sigma(x, t) & =\varepsilon(x, t)+b_{0} \mathrm{D}_{t}^{\alpha} \varepsilon(x, t), \\
\varepsilon(x, t) & =\mathcal{E}_{x}^{\beta} u(x, t),
\end{aligned}
$$

where $\alpha \in[0,1), \beta \in(0,1),{ }_{0} \mathrm{D}_{t}^{\alpha}$ is the fractional differential operator, defined as follows. Let $t, \gamma \in \mathbb{R}$ and $H$ denote the Heaviside function. Then one defines

$$
f_{\gamma}(t)= \begin{cases}\frac{t^{\gamma-1}}{\Gamma(\gamma)} H(t), & \gamma>0, \\ \frac{\mathrm{d}^{N}}{\mathrm{~d} t^{N}} f_{\gamma+N}(t), & \gamma \leq 0, \gamma+N>0, N \in \mathbb{N}\end{cases}
$$

and for $g \in \mathcal{S}^{\prime}$, with support in the region $t>0$,

$$
{ }_{0} \mathrm{D}_{t}^{\gamma} g=f_{-\gamma} *_{t} g=\frac{\mathrm{d}}{\mathrm{d} t} f_{1-\gamma} *_{t} g .
$$

Upon Fourier transform we may solve (8) with respect to $\sigma$ by

$$
\sigma(x, t)=\mathcal{F}_{\tau \rightarrow t}^{-1}\left[\frac{1+b \mathrm{e}^{\mathrm{i} \frac{\alpha \pi}{2}}(\tau-\mathrm{i} 0)^{\alpha}}{1+a \mathrm{e}^{\mathrm{i} \frac{\alpha \pi}{2}}(\tau-\mathrm{i} 0)^{\alpha}}\right] *_{t} \varepsilon(x, t) .
$$

Indeed, by [5, Example 7.1.17], $\widehat{f_{1-\alpha}}=\mathrm{e}^{-\mathrm{i} \frac{(1-\alpha) \pi}{2}}(\tau-\mathrm{i} 0)^{\alpha-1}$, implying also $\widehat{f_{-\alpha}}=\mathcal{F}\left(\frac{d}{d t} f_{1-\alpha}\right)=$ $\mathrm{i} \tau \mathrm{e}^{-\mathrm{i} \frac{(1-\alpha) \pi}{2}}(\tau-\mathrm{i} 0)^{\alpha-1}=\mathrm{e}^{\mathrm{i} \frac{\alpha \pi}{2}}(\tau-\mathrm{i} 0)^{\alpha}$, hence we find that (10) solves (8) . Finally, inserting this 
into (7) and observing (9), we arrive at Equation (5). As in 11, we study the Cauchy problem (55)6 with distributional initial values in the form (1).

In Section 2 we analyze the microlocal properties of the space-fractional wave equation and in Section 3 we address an analogue of the non-characteristic regularity of solutions to the timefractional Zener wave equation.

\section{The space-fractional wave equation}

We consider the solution for the special case of problem (11) with $u_{0}, v_{0} \in \mathcal{E}^{\prime}(\mathbb{R})$ when $\alpha=0$ and $0<\beta<1$, which leads to the so-called space-fractional wave equation

$$
Z u(x, t)=\partial_{t}^{2} u(x, t)-\partial_{x} \mathcal{E}_{x}^{\beta} u(x, t)=u_{0}(x) \otimes \delta^{\prime}(t)+v_{0}(x) \otimes \delta(t) .
$$

With $b_{\beta}:=\sqrt{\sin \frac{\beta \pi}{2}}$ we have the solution $u$ with $\operatorname{supp}(u) \subseteq\{t \geq 0\}$ in the form (cf. [1] $)$

$$
u=u_{0} *_{x} \underbrace{\mathcal{F}_{\xi \rightarrow x}^{-1}\left[\cos \left(b_{\beta}|\xi|^{\frac{1+\beta}{2}} t\right) H(t)\right]}_{E_{0}}+v_{0} *_{x} \underbrace{\mathcal{F}_{\xi \rightarrow x}^{-1}\left[\frac{\sin \left(b_{\beta}|\xi|^{\frac{1+\beta}{2}} t\right)}{b_{\beta}|\xi|^{\frac{1+\beta}{2}}} H(t)\right]}_{E_{1}} .
$$

Remark 2.1. (i) We observe that $E_{1}$ is a fundamental solution of $Z$, i.e., $Z E_{1}=\delta$. Furthermore, the equations $E_{0}=\partial_{t} E_{1}$ and $Z E_{0}(x, t)=\delta(x) \otimes \delta^{\prime}(t)$ hold on $\mathbb{R}^{2}$.

Furthermore, both $E_{0}$ and $E_{1}$ are weakly smooth with respect to $t$ when $t \neq 0$. We note that $t \mapsto E_{1}(t)$ is continuous $\mathbb{R} \rightarrow \mathcal{S}^{\prime}(\mathbb{R})$ with $E_{1}(0)=0$, whereas $\lim _{t \rightarrow 0+} E_{0}(t)=\delta \neq 0=$ $\lim _{t \rightarrow 0-} E_{0}(t) ; E_{0}$ is weakly measurable with respect to $t \in \mathbb{R}$.

(ii) It is apparent from (12) and the assumption $u_{0}, v_{0} \in \mathcal{E}^{\prime}(\mathbb{R})$ that the partial $x$-Fourier transform $\mathcal{F}_{x \rightarrow \xi}(u)$ of $u$ is a continuous function with respect to $\xi$ and of moderate growth. Hence the multiplication $\widehat{g_{\gamma}} \cdot \mathcal{F}_{x \rightarrow \xi}(u)$ with $\widehat{g_{\gamma}}(\xi):=|\xi|^{\gamma}(-1<\gamma<1)$ gives a locally integrable function of moderate growth with respect to $\xi$, and $G_{\gamma} u:=\mathcal{F}_{\xi \rightarrow x}^{-1}\left(\widehat{g}_{\gamma} \cdot \mathcal{F}_{x \rightarrow \xi}(u)\right)$ is defined in $\mathcal{S}^{\prime}\left(\mathbb{R}^{2}\right)$. The same is true, if in place of $u$ we consider $\tilde{u}=\mathcal{F}_{\xi \rightarrow x}^{-1}\left(\exp \left(i b_{\beta}|\xi|^{\frac{1+\beta}{2}} t\right)\right) *_{x} u_{0}$ and other similarly constructed distributions, e.g., $w=G_{\sigma} \tilde{u}$. We will repeatedly make use of this fact within the current section in course of the following proofs.

(iii) For fixed $t>0$, the linear operators of convolution with $E_{0}(t)$ and $E_{1}(t)$ are bounded $L^{p}(\mathbb{R}) \rightarrow L^{p}(\mathbb{R})$, if $1<p<\infty$, by Hörmander's multiplier theorem, since their Fourier transforms are in $L^{\infty}(\mathbb{R}) \cap C^{1}(\mathbb{R} \backslash\{0\})$ with derivative bounded by a constant times $|\xi|^{-1}$ (cf. 3, Corollary 8.11] or [5, Theorem 7.9.5]).

Lemma 2.2. For $j=0,1$, let $E_{j}^{+}$denote the restriction of $E_{j}$ to the open half-space $\{t>0\}$. Then the wave front sets are given by

$$
\mathrm{WF}\left(E_{0}^{+}\right)=\mathrm{WF}\left(E_{1}^{+}\right)=\{(0, t ; \xi, 0) \mid t>0, \xi \neq 0\}=: W_{0} .
$$

Proof. From $\partial_{t} E_{1}^{+}=E_{0}^{+}$we immediately deduce

$$
\mathrm{WF}\left(E_{0}^{+}\right) \subseteq \mathrm{WF}\left(E_{1}^{+}\right) \subseteq \mathrm{WF}\left(E_{0}^{+}\right) \cup\{(x, t ; \xi, 0) \mid t>0, \xi \neq 0\},
$$

where the right-most set corresponds to the characteristic set of $\partial_{t}$ when considered as partial differential operator on $\mathbb{R} \times] 0, \infty[$. 
Claim 1: $\operatorname{Both}, \operatorname{WF}\left(E_{0}^{+}\right)$and $\operatorname{WF}\left(E_{1}^{+}\right)$, are contained in $W_{0}$.

Let $t>0$, put $e_{0}^{+}(\xi, t):=\cos \left(b_{\beta}|\xi|^{\frac{1+\beta}{2}} t\right)$ and $e_{1}^{+}(\xi, t):=\sin \left(b_{\beta}|\xi|^{\frac{1+\beta}{2}} t\right) /\left(b_{\beta}|\xi|^{\frac{1+\beta}{2}}\right)$, and choose $\rho \in C^{\infty}(\mathbb{R})$ such that $\rho(\xi)=0$ for $|\xi| \leq 1 / 2$ and $\rho(\xi)=1$ for $|\xi| \geq 1$. Then at fixed arbitrary $t>0$ and $j \in\{0,1\}$ we have

$$
E_{j}^{+}(t)=\mathcal{F}_{\xi \rightarrow x}^{-1}\left(e_{j}^{+}(\xi, t)\right)=\mathcal{F}_{\xi \rightarrow x}^{-1}\left(e_{j}^{+}(\xi, t) \rho(\xi)\right)+\mathcal{F}_{\xi \rightarrow x}^{-1}\left(e_{j}^{+}(\xi, t)(1-\rho(\xi))\right)=: F_{j, 1}(t)+F_{j, 2}(t) .
$$

We observe that $(\xi, t) \mapsto e_{j}^{+}(\xi, t)(1-\rho(\xi))$ is continuous, has compact $\xi$-support, and is smooth with respect to $t$, more precisely, $e_{j}^{+}(1-\rho) \in C^{\infty}(] 0, \infty\left[, C_{\mathrm{c}}(\mathbb{R})\right)$, hence by linearity, commutativity with $\frac{d}{d t}$, and continuity of the inverse Fourier transform with respect to $\xi$, we have $F_{j, 2} \in$ $C^{\infty}(] 0, \infty\left[, \mathcal{F}^{-1}\left(C_{\mathrm{c}}(\mathbb{R})\right)\right) \subseteq C^{\infty}(] 0, \infty\left[, C^{\infty}(\mathbb{R})\right) \subseteq C^{\infty}(] 0, \infty[\times \mathbb{R})$, thus, $\operatorname{WF}\left(E_{j}^{+}\right)=\operatorname{WF}\left(F_{j, 1}\right)$.

Note that $a_{j}(x, t, \xi):=e_{j}^{+}(\xi, t) \rho(\xi)$ define functions in $C^{\infty}\left(\mathbb{R}^{2} \times \mathbb{R}\right)(j=0,1)$ and that $a_{1}(x, t, \xi)=\int_{0}^{t} a_{0}(x, s, \xi) d s$. Furthermore, $a_{0}$ is a symbol of class $S_{\frac{1-\beta}{2}}^{0}, \frac{1+\beta}{2}\left(\mathbb{R}^{2} \times \mathbb{R}\right)$, since $e_{0}^{+}$is the real part of a function of a special case of the type discussed in [4, Example 1.1.5.] (with appropriate choices of parameters and variable names); here, the condition $0 \leq \beta<1$ is crucial. In fact, the corresponding symbol estimates need only be carried out in the region $|\xi|>1$ and are elementary in our case. We also see directly that $x$-derivatives of $a_{1}$ vanish, any $\xi$-derivative of $a_{1}$ and $a_{0}$ - as well as $a_{1}$ and $a_{0}$ themselves - have essentially the same bounds when $(x, t)$ vary in a compact subset and $|\xi|>1$; furthermore, any $t$-derivative of $a_{1}$ brings us back to estimating $a_{0}$ with one order less in the $t$-derivatives, thus $a_{1}$ is contained in the same symbol class as $a_{0}$.

To complete the proof of Claim 1 , we observe that $F_{j, 1}(j=0,1)$, being an inverse Fourier transform, can be written as oscillatory integral (in the sense of [5, Theorem 7.8.2]) with symbol $a_{j}(x, t, \xi) /(2 \pi)$ and phase funtion $\phi(x, t, \xi)=x \xi$ in both cases. Thus, according to [5, Theorem 8.1.9], the only contributions to the wave front sets can stem from points with stationary phase, i.e.,

$$
\begin{aligned}
W F\left(E_{j}^{+}\right) & \subseteq\left\{\left(x, t ; \partial_{x} \phi(x, t, \xi), \partial_{t} \phi(x, t, \xi)\right) \mid t>0, \exists \xi \neq 0: \partial_{\xi} \phi(x, t, \xi)=0\right\} \\
& =\{(0, t, \xi, 0) \mid t>0, \xi \neq 0\}=W_{0} .
\end{aligned}
$$

Claim 2: $\quad W_{0} \subseteq \mathrm{WF}\left(E_{0}^{+}\right)$.

Note that due to the symmetry of $\mathcal{F}\left(E_{0}^{+}\right)$with respect to $\xi \mapsto-\xi$ and the result of Claim 1 we have $(0, t ; \xi, 0) \in \mathrm{WF}\left(E_{0}^{+}\right) \Leftrightarrow(0, t ;-\xi, 0) \in \mathrm{WF}\left(E_{0}^{+}\right) \Leftrightarrow(0, t) \in \operatorname{singsupp}\left(E_{0}^{+}\right)$. Thus, it suffices to show that $E_{0}^{+}$is nonsmooth along the half axis $x=0, t>0$.

We introduce $\widetilde{E}(t):=\mathcal{F}_{\xi \rightarrow x}^{-1}\left[\exp \left(i b_{\beta} t|\xi|^{\frac{1+\beta}{2}}\right)\right]$ and observe that by the elementary relations $\cos (z)=(\exp (i z)+\overline{\exp (i z)}) / 2$ and $\mathcal{F}^{-1}(\bar{v})(x)=\overline{\mathcal{F}^{-1}(v)(-x)}$ and employing the ad-hoc notation $R^{*}$ for the pull-back by $R(x, t)=(-x, t)$ we may write

$$
E_{0}^{+}=\frac{1}{2}\left(\widetilde{E}+\overline{R^{*} \widetilde{E}}\right) .
$$

Since we are here concerned solely with the question of smoothness at the points $(0, t)$ for any $t>0$ and $t \mapsto E_{0}^{+}(t)$ as well as $t \mapsto \widetilde{E}(t)$ is smooth, we may note: $E_{0}^{+}$is non-smooth at $(0, t) \Leftrightarrow$ $E_{0}^{+}(t)$ is non-smooth at $x=0 \Leftrightarrow \widetilde{E}(t)$ is non-smooth at $x=0$.

Let $f:=\mathcal{F}_{\xi \rightarrow x}^{-1}\left[\mathrm{e}^{\mathrm{i} b_{\beta}|\xi|^{\sigma}}\right] \in \mathcal{S}^{\prime}(\mathbb{R})$, abbreviating $\sigma=(1+\beta) / 2$, and observe that for $t>0$, rescaling by $\xi \mapsto \xi t^{1 / \sigma}$ on the Fourier transformed side, we have $\widetilde{E}(t)=f\left(. / t^{1 / \sigma}\right) / t^{1 / \sigma}$ and therefore may reduce the question of smoothness of $\tilde{E}(t)$ at $x=0$ further to that of smoothness of $f$ at zero. 
Choose $\eta \in C^{\infty}(\mathbb{R})$ such that $\eta=0$ near $\xi=0, \eta=1$ for $|\xi|>1$, and write $\hat{f}=(1-\eta) \hat{f}+\eta \hat{f}$. Since $(1-\eta) \hat{f} \in C_{c}(\mathbb{R})$, smoothness of $f$ is equivalent to that of $\mathcal{F}^{-1}(\eta \hat{f})$.

Let $\theta>0$ and put

$$
Q_{\theta} w:=\mathcal{F}_{\xi \rightarrow x}^{-1}\left[\frac{\eta(\xi)}{|\xi|^{\theta}}\right] * w \text { and } m_{\theta}(\xi):=\mathcal{F}_{x \rightarrow \xi}\left[Q_{\theta} f\right](\xi)=\frac{\eta(\xi)}{|\xi|^{\theta}} \mathrm{e}^{\mathrm{i} b_{\beta}|\xi|^{\sigma}} .
$$

Here, $Q_{\theta}$ is a pseudodifferential operator with symbol $q_{\theta}(x, \xi)=\eta(\xi) /|\xi|^{\theta}$, which clearly satisfies $\left|q_{\theta}(\xi)\right|=1 /|\xi|^{\theta}$, if $|\xi|>1$, hence $Q_{\theta}$ is elliptic (of order $-\theta$ ). Thus, smoothness of $f$ at 0 turns out to be equivalent to smoothness of $Q_{\theta} f$ at 0 . Note that $Q_{\theta} f$ is smooth off 0 by essentially the same arguments used as with $E_{0}$ and the symbol $a_{0}$ in Claim 1.

The non-smoothness of $Q_{\theta} f$ at 0 is shown thanks to an asymptotic result by G. H. Hardy mentioned in [10, p. 357, 5.3(ii)], with parameters $a, b$ there to be identified with $\sigma, \theta$ respectively; note that $1>\sigma=(1+\beta) / 2>1 / 2$ and let us suppose that $\theta>\left(1-\frac{\sigma}{2}\right) /(1-\sigma)>0$; then we conclude that there is some constant $c_{2}>0$ such that

$$
\left(Q_{\theta} f\right)(x)=c_{1} \mathrm{e}^{\mathrm{i} \frac{c_{2}}{|x|^{\alpha}}} \frac{1}{|x|^{\gamma}}+O\left(\frac{1}{|x|^{\gamma-\frac{\alpha}{2}}}\right) \quad(x \rightarrow 0),
$$

where $\alpha=\frac{\sigma}{1-\sigma}$ and $\gamma=\frac{\theta(1-\sigma)-1+\frac{\sigma}{2}}{2 \sigma-1}>0$. Thus, $Q_{\theta} f(x)$ cannot be bounded as $x \rightarrow 0$ and therefore cannot be continuous near $x=0$, which completes the proof of Claim 2 .

From Claims 1 and 2 in conjunction with the first inclusion relation in $(*)$ established at the beginning of the proof, we obtain

$$
W_{0} \subseteq \mathrm{WF}\left(E_{0}^{+}\right) \subseteq \mathrm{WF}\left(E_{1}^{+}\right) \subseteq W_{0},
$$

which implies that equality holds throughout and completes the proof.

Based on the results of Lemma 2.2 we will investigate the influence of the singularities in the initial data $u_{0}$ and $v_{0}$ on the wave front set of the solution $u$ to (11). We emphasize that the proof of Theorem 2.4 below uses only the inclusion relation $\mathrm{WF}\left(E_{j}^{+}\right) \subseteq W_{0}$ in its first part and provides an independent, more general, proof of equality in this relation-thus substituting the argument of Claim 2 above drawing on Hardy's asymptotics by advanced microlocal techniques.

Remark 2.3. If $v_{0}=0$ and $t>0$ the solution formula (12) simply means $u^{+}(t)=E_{0}^{+}(t) * u_{0}$. Since $\operatorname{singsupp}\left(E_{0}^{+}(t)\right)=\{0\}$ a smooth cut-off $\rho$ near $x=0$ implies $\left(E_{0}^{+}(t)(1-\rho)\right) * u_{0} \in$ $C^{\infty}(\mathbb{R})$, hence it suffices to investigate the singularity structure of $\left(E_{0}^{+}(t) \rho\right) * u_{0}$, where now both convolution factors belong to $\mathcal{E}^{\prime}(\mathbb{R})$. At fixed $t$, this enables us to employ the methods and results from [6, Section 16.3] on singular supports of convolutions (or to extend these techniques to wave front sets as suggested by Hörmander directly after the statement of [6. Definition 16.3.2]). Though a bit technical, it is not difficult to see that one will then obtain equality of the closed convex hulls of $\operatorname{singsupp}\left(u^{+}(t)\right)$ and $\operatorname{singsupp}\left(u_{0}\right)$. However, even having information on $\mathrm{WF}\left(u^{+}(t)\right)$ for every $t>0$ would not yield precise microlocal information on $\operatorname{WF}\left(u^{+}\right)$in terms of the two-dimensional directions in the cotangent fiber.

Theorem 2.4. Let $u_{0}, v_{0} \in \mathcal{E}^{\prime}(\mathbb{R})$ and denote by $u^{+}$the restriction of the solution $u$ to (11) to the half-space of future time $\mathbb{R} \times] 0, \infty\left[\right.$, then $\mathrm{WF}\left(u^{+}\right)$is invariant under translations $(x, t) \mapsto(x, t+s)$ with $s>0$ and

$$
\mathrm{WF}\left(u^{+}\right) \subseteq\left\{(x, t ; \xi, 0) \mid t>0,(x, \xi) \in W F\left(u_{0}\right) \text { or }(x, \xi) \in W F\left(v_{0}\right)\right\} .
$$


Moreover, in case $v_{0}$ is smooth we have the more precise statement

$$
\mathrm{WF}\left(u^{+}\right)=\left\{(x, t ; \xi, 0) \mid t>0,(x, \xi) \in W F\left(u_{0}\right)\right\},
$$

and similarly $\mathrm{WF}\left(u^{+}\right)=\left\{(x, t ; \xi, 0) \mid t>0,(x, \xi) \in W F\left(v_{0}\right)\right\}$, if $u_{0}$ is smooth.

To prepare for the proof of the theorem we need a technical lemma on "symbol corrections".

Lemma 2.5. Let $\sigma \in(0,1)$ and $y(\xi, \tau)=-\tau+b_{\beta}|\xi|^{\sigma}$. Let $\Gamma \subseteq \mathbb{R}^{2}$ (representing the $(\xi, \tau)$ plane) be the union of a closed disc around $(0,0)$ and a closed narrow cone containg the $\tau$-axis and being symmetric with respect to both axes. Let $\Gamma^{\prime}$ be a closed set of the same shape as $\Gamma$, but with slightly larger disc and opening angle of the cone. Let $\tilde{b} \in S^{0}\left(\mathbb{R}^{2} \times \mathbb{R}^{2}\right)$ such that $\tilde{b}(x, t, \xi, \tau)$ is real, constant with respect to $(x, t)$, homogenous of degree 0 with respect to $(\xi, \tau)$ away from the disc contained in $\Gamma^{\prime}$, and such that $\tilde{b}(x, t, \xi, \tau)=0$, if $(\xi, \tau) \in \Gamma, \tilde{b}(x, t, \xi, \tau)=1$, if $(\xi, \tau) \notin \Gamma^{\prime}$. Then $y \tilde{b}$ is a symbol belonging to the class $S^{1}\left(\mathbb{R}^{2} \times \mathbb{R}^{2}\right)$.

Proof. By construction of $\tilde{b}$, it suffices to check the symbol estimates when $(\xi, \tau)$ is outside $\Gamma$, say $|\tau|<c|\xi|$, and $|\xi|+|\tau|$ is large. The upper bound in order zero is clear from $|y \tilde{b}| \leq$ $C_{0}\left(|\xi|^{\sigma}+|\tau|\right) \leq C_{0}^{\prime}(1+|\xi|+|\tau|)$. A derivative $\partial_{\xi}^{\alpha_{1}} \partial_{\tau}^{\alpha_{2}}(y \tilde{b})$ with $\alpha_{1}+\alpha_{2}=n \geq 1$ involves (apart from combinatorial constants) only nonzero terms of the form $\partial_{\tau} y \partial_{\xi}^{l} \partial_{\tau}^{m-1} \tilde{b}=-\partial_{\xi}^{l} \partial_{\tau}^{m-1} \tilde{b}$ with $l+m=n$ or $\partial_{\xi}^{k} y \partial_{\xi}^{l} \partial_{\tau}^{m} \tilde{b}$ with $k+l+m=n$, hence it suffices to estimate these. We have $\left|\partial_{\xi}^{l} \partial_{\tau}^{m-1} \tilde{b}(\xi, \tau)\right| \leq C_{l, m}(1+|\xi|+|\tau|)^{0-l-(m-1)}=C_{l, m}(1+|\xi|+|\tau|)^{1-n}$ and

$$
\begin{aligned}
\left|\partial_{\xi}^{k} y(\xi, \tau) \partial_{\xi}^{l} \partial_{\tau}^{m} \tilde{b}(\xi, \tau)\right| & =C^{\prime}|\xi|^{\sigma-k}\left|\partial_{\xi}^{l} \partial_{\tau}^{m} \tilde{b}(\xi, \tau)\right| \leq \tilde{C} \frac{(1+|\xi|+|\tau|)^{-l-m}}{1+|\xi|^{k-\sigma}} \\
& \leq \tilde{C} \frac{(1+|\xi|+|\tau|)^{-l-m}}{1+\frac{1}{2}|\xi|^{k-1}+\frac{1}{2}|\xi|^{k-1}} \leq \tilde{C} \frac{(1+|\xi|+|\tau|)^{-l-m}}{1+\frac{1}{2}|\xi|^{k-1}+\frac{1}{2 c}|\tau|^{k-1}} \\
& \leq C(1+|\xi|+|\tau|)^{1-k-l-m}=C(1+|\xi|+|\tau|)^{1-n} .
\end{aligned}
$$

Proof of the Theorem. We consider the case when $v_{0}$ is smooth, which we may immediately reduce to $v_{0}=0$, since the contribution of $E_{1}^{+} *_{x} v_{0}$ to the solution is smooth. Put $K=f^{*} E_{0}^{+}$, where $f: \mathbb{R} \times] 0, \infty[\times \mathbb{R} \rightarrow \mathbb{R} \times] 0, \infty\left[=: \Omega\right.$ is given by $f(x, t, y)=(x-y, t)$, and $f^{*}$ is the distributional pull-back in the sense of [5, Theorem 6.1.2]. Then $K \in \mathcal{D}^{\prime}(\Omega \times \mathbb{R})$ and [5], Theorem 8.2.4] and Lemma 2.2 imply

$$
\mathrm{WF}(K) \subseteq\{(x, t, y ; \xi, 0,-\xi) \mid x=y, \xi \neq 0\}=\{(x, t, x) \mid x, t \in \mathbb{R}\} \times\{(\xi, 0,-\xi) \mid \xi \neq 0\} .
$$

We have $u^{+}=\left.u\right|_{\{t>0\}}=u_{0} *_{x} E_{0}^{+}$, whose action on test functions $\varphi \in \mathcal{D}(\Omega)$ can be written in the form $\left\langle u^{+}, \varphi\right\rangle=\left\langle K, \varphi \otimes u_{0}\right\rangle$, if $u_{0} \in \mathcal{D}(\mathbb{R})$, i.e., $u_{0} \mapsto u^{+}$is the linear map $S: \mathcal{D}(\mathbb{R}) \rightarrow \mathcal{D}^{\prime}(\Omega)$ with distribution kernel $K$. Since $\mathrm{WF}^{\prime}(K)_{\mathbb{R}}=\{(y, \eta) \mid \exists(x, t):(x, t, y ; 0,0,-\eta) \in \mathrm{WF}(K)\}=\emptyset$, [5. Theorem 8.2.13] implies that $S$ may be extended to a map $\mathcal{E}^{\prime}(\mathbb{R}) \rightarrow \mathcal{D}^{\prime}(\Omega)$ and satisfies

$$
\mathrm{WF}\left(S u_{0}\right) \subseteq \mathrm{WF}(K)_{\Omega} \cup \mathrm{WF}^{\prime}(K) \circ \mathrm{WF}\left(u_{0}\right),
$$

where $\mathrm{WF}(K)_{\Omega}=\{(x, t ; \xi, \tau) \mid(x, t, y ; \xi, \tau, 0) \in \mathrm{WF}(K)$ for some $y \in \mathbb{R}\}=\emptyset$ and $\mathrm{WF}^{\prime}(K)=$ $\{(x, t, y ; \xi, \tau, \eta) \mid(x, t, y ; \xi, \tau,-\eta) \in W F(K)\}=\{(x, t, x ; \xi, 0, \xi) \mid t>0, \xi \neq 0\}$. Thus, we obtain

$$
\begin{aligned}
\mathrm{WF}\left(u^{+}\right) & \subseteq\left\{(x, t ; \xi, \tau) \mid \exists(y, \eta) \in \mathrm{WF}\left(u_{0}\right):(x, t, y ; \xi, \tau, \eta) \in \mathrm{WF}^{\prime}(K)\right\} \\
& \subseteq\left\{(x, t ; \xi, \tau) \mid t>0, \exists(y, \eta) \in W F\left(u_{0}\right): y=x, \tau=0, \eta=\xi\right\}
\end{aligned}
$$


i.e.,

$$
\mathrm{WF}\left(u^{+}\right) \subseteq\left\{(x, t ; \xi, 0) \mid(x, \xi) \in \mathrm{WF}\left(u_{0}\right), t>0\right\},
$$

and the remaining part of the proof is concerned with showing that equality holds in (13).

As in the proof of Lemma 2.2 let $\tilde{E}(t):=\mathcal{F}_{\xi \rightarrow x}^{-1}\left[\exp \left(i b_{\beta} t|\xi|^{\sigma}\right)\right]$ with $\sigma:=(1+\beta) / 2$, but this time for any $t \in \mathbb{R}$, and put $\tilde{u}(t):=\tilde{E}(t) * u_{0}$. We have $D_{t} \widehat{\widetilde{E}(t)}=\frac{1}{i} \partial_{t} \widehat{\widetilde{E}(t)}=b_{\beta}|\xi|^{\sigma} \mathrm{e}^{\mathrm{i} b_{\beta}|\xi|^{\sigma} t}=$ $b_{\beta}|\xi|^{\sigma} \widehat{\tilde{E}(t)}$, which implies

$$
Y \tilde{E}:=-D_{t} \tilde{E}+A_{x}^{\sigma} \tilde{E}=0, \quad \tilde{E}(0)=\delta,
$$

where $A_{x}^{\sigma} w=\mathcal{F}_{\xi \rightarrow x}^{-1}\left[b_{\beta}|\xi|^{\sigma}\right] *_{x} w$ (with $w$ of the type as in Remark 2.1(ii)). Moreover, $\tilde{u}$ solves the initial value problem

$$
Y \tilde{u}=\left(-D_{t}+A_{x}^{\sigma}\right) \tilde{u}=\left(-D_{t} \tilde{E}+A_{x}^{\sigma} \tilde{E}\right) *_{x} u_{0}=0, \quad \tilde{u}(0)=u_{0} .
$$

Note that, since $b_{\beta}^{2}|\xi|^{2 \sigma}=\sin (\beta \pi / 2)|\xi|^{\beta+1}$ is precisely the "symbol" of $-\mathcal{E}_{x}^{\beta} \partial_{x}$, we have a (commutative) factorization of $Z$ by $\left(D_{t}+A_{x}^{\sigma}\right)\left(-D_{t}+A_{x}^{\sigma}\right) v=-D_{t}^{2} v+A_{x}^{\sigma} A_{x}^{\sigma} v=\partial_{t}^{2} v+$ $\mathcal{F}_{\xi \rightarrow x}^{-1}\left(b_{\beta}^{2}|\xi|^{2 \sigma}\right) *_{x} v=Z v$, i.e., $Z=\bar{Y} \cdot Y$, where we have put $\bar{Y}:=D_{t}+A_{x}^{\sigma}$.

Before entering the detailed microlocal analysis of $\tilde{u}$ let us anticipate its relevance for $u^{+}$: We will show in Equation (17) below, that in the region with $t>0, \tilde{u}$ provides a "lower bound" for the wave front set of $u^{+}$, in fact, we will show equality of the wave front sets at the end of the proof.

In studying the propagation of singularities for problem $(*)$ we encounter the nuisance that $y(\xi, \tau)=-\tau+b_{\beta}|\xi|^{\sigma}$ is not quite a symbol of order 1 in $\xi$ and $\tau$, since $y$ is nonsmooth at zero and, furthermore, the symbol estimates obviously fail, e.g., for $\left|\partial_{\xi}^{2} y(\xi, \tau)\right|=\left.|\sigma(\sigma-1)| \xi\right|^{\sigma-2} \mid$ when $\tau \rightarrow \infty$, there would have to be a bound of decrease $(1+|\tau|+|\xi|)^{-1}$ for large $|\tau|+|\xi|$. A remedy of this second kind of "symbol failure" is discussed in [8, Theorem 18.1.35], see also a comment below [8, Theorem 23.1.4], which we essentially follow in studying the propagation of singularities for $\tilde{u}$ considered as solution to

$$
Y B \tilde{u}=B Y \tilde{u}=0, \quad \tilde{u}(0)=u_{0},
$$

where $B=$ op $(\tilde{b}) \in \Psi^{0}\left(\mathbb{R}^{2}\right)$ is the pseudodifferential operator associated with a symbol $\tilde{b}$ given as in Lemma 2.5. Thus, $Y B=$ op $(y \tilde{b}) \in \Psi^{1}\left(\mathbb{R}^{2}\right)$ has principal symbol

$$
q(\xi, \tau):=-\tau \tilde{b}(\xi, \tau),
$$

which is real and homogeneous of degree one, and, modulo a regularizing contribution, can be considered properly supported.

By [7, Theorem 26.1.1] $\mathrm{WF}(\tilde{u})$ is invariant under the flow corresponding to the Hamiltonian vector field

$$
H_{q}(x, t, \xi, \tau)=\left(\begin{array}{c}
-\partial_{\xi} q \\
-\partial_{\tau} q \\
\partial_{x} q \\
\partial_{t} q
\end{array}\right)=\left(\begin{array}{c}
\tau \partial_{\xi} \tilde{b} \\
\tilde{b}+\tau \partial_{\tau} \tilde{b} \\
0 \\
0
\end{array}\right)
$$

and is contained in the characteristic set $\operatorname{Char}(Y B)=\mathbb{R}^{2} \times\{(\xi, 0) \mid \xi \neq 0\}$, i.e., $\operatorname{WF}(\tilde{u}) \subseteq$ Char $(Y B)$ In fact, a refinement of the latter inclusion relation is available, since $\tilde{u}=\tilde{E} *_{x} u_{0}$ and we may argue very similar to proof of (13), noting (as in Claim 2 of the proof of Lemma 2.2) that $\tilde{E}$ is microlocally equivalent to $\mathcal{F}_{\xi \rightarrow x}\left(\cos \left(b_{\beta}|\xi|^{\sigma} t\right)\right)$, and deduce

$$
\mathrm{WF}(\tilde{u}) \subseteq\left\{(x, t ; \xi, 0) \mid(x, \xi) \in \mathrm{WF}\left(u_{0}\right), t \in \mathbb{R}\right\} .
$$


Solving the Hamiltonian equations

$$
\begin{aligned}
& \dot{x}=\tau \partial_{\xi} \tilde{b}(\xi, \tau), \quad \dot{t}=\tilde{b}(\xi, \tau)+\tau \partial_{\tau} \tilde{b}(\xi, \tau), \quad \dot{\xi}=0, \quad \dot{\tau}=0, \\
& \text { with }(x(0), t(0), \xi(0), \tau(0))=\left(x_{0}, t_{0}, \xi_{0}, 0\right) \in \operatorname{Char}(Y B),
\end{aligned}
$$

we obtain $\forall s \in \mathbb{R}: x(s)=x_{0}, t(s)=t_{0}+s \tilde{b}\left(\xi_{0}, 0\right), \xi(s)=\xi_{0}, \tau(s)=0$. We may suppose that $\tilde{b}\left(\xi_{0}, 0\right)=1$, since $\xi_{0} \neq 0$ and characteristic sets as well as wave front sets are conic with respect to the cotangent fibers. Hence the bicharacteristic flow evolves along the $t$-direction with fixed cotangent directions $\left(\xi_{0}, 0\right)$ only. Therefore we have $\left(x_{0}, t_{0} ; \xi_{0}, 0\right) \in \mathrm{WF}(\tilde{u})$ if and only if $\left(x_{0}, 0 ; \xi_{0}, 0\right) \in \mathrm{WF}(\tilde{u})$. We claim that the latter is in turn equivalent to $\left(x_{0}, \xi_{0}\right) \in \mathrm{WF}\left(u_{0}\right)$, from which, together with (14), we may then conclude

$$
\mathrm{WF}(\tilde{u})=\left\{(x, t ; \xi, 0) \mid(x, \xi) \in \mathrm{WF}\left(u_{0}\right), t \in \mathbb{R}\right\} .
$$

We have claimed: $\left(x_{0}, 0 ; \xi_{0}, 0\right) \in \mathrm{WF}(\tilde{u}) \Leftrightarrow\left(x_{0}, \xi_{0}\right) \in W F\left(u_{0}\right)$.

The implication ' $\Rightarrow$ ' follows from (14). For the converse, note that, according to [5, Theorem 8.2.4] and the fact that $\mathrm{WF}(\tilde{u}) \subseteq \operatorname{Char}(Y B)$ contains no directions $(0, \tau)$ in the fiber, we may write $\tilde{u}(t)=f_{t}^{*} \tilde{u}$ for any $t \in \mathbb{R}$, where $f_{t}(x)=(x, t)$ as a map $\mathbb{R} \rightarrow \mathbb{R}^{2}$, and obtain

$$
\mathrm{WF}(\tilde{u}(t)) \subseteq f_{t}^{*} \mathrm{WF}(\tilde{u})=\{(x, \xi) \mid(x, t ; \xi, 0) \in \mathrm{WF}(\tilde{u})\}=\{(x, \xi) \mid(x, 0 ; \xi, 0) \in \mathrm{WF}(\tilde{u})\},
$$

where the last equality follows from the Hamiltonian invariance proven above. In particular, when $t=0$ we have $\tilde{u}(0)=u_{0}$, so that $\mathrm{WF}\left(u_{0}\right)=\mathrm{WF}(\tilde{u}(0)) \subseteq\{(x, \xi) \mid(x, 0 ; \xi, 0) \in \mathrm{WF}(\tilde{u})\}$, which proves the part ' $\Leftarrow$ ' of the claim and thus establishes (15).

We are now ready to clarify the microlocal relation between $\tilde{u}$ and $u^{+}$: In the subdomain with $t>0$ we have

$$
\bar{Y} u^{+}=\left(D_{t} E_{0}^{+}+A_{x}^{\sigma} E_{0}^{+}\right) *_{x} u_{0}=A_{x}^{\sigma} \tilde{E} *_{x} u_{0}=A_{x}^{\sigma} \tilde{u}
$$

since $\mathcal{F}_{x \rightarrow \xi}\left[D_{t} E_{0}^{+}(t)+A_{x}^{\sigma} E_{0}^{+}(t)\right]=-i \partial_{t}\left(\cos \left(b_{\beta}|\xi|^{\sigma} t\right)\right)+b_{\beta}|\xi|^{\sigma} \cos \left(b_{\beta}|\xi|^{\sigma} t\right)=b_{\beta}|\xi|^{\sigma} \widehat{\tilde{E}(t)}$.

Denoting by $\tilde{u}^{+}$the restriction of $\tilde{u}$ to the half-plane of positive time we claim that the following two inclusions hold:

$$
\text { (I) } \operatorname{WF}\left(\bar{Y} u^{+}\right) \subseteq \mathrm{WF}\left(u^{+}\right), \text {and }(\mathrm{II}) \operatorname{WF}\left(\tilde{u}^{+}\right) \subseteq \mathrm{WF}\left(A_{x}^{\sigma} \tilde{u}^{+}\right) .
$$

Since $\bar{Y}=D_{t}+A_{x}^{\sigma}$ and $D_{t}$ clearly is a microlocal, i.e, $\operatorname{WF}\left(D_{t} w\right) \subseteq \operatorname{WF}(w)$ for any $w \in \mathcal{D}^{\prime}\left(\mathbb{R}^{2}\right)$, we may reduce (I) to the statement $\operatorname{WF}\left(A_{x}^{\sigma} u^{+}\right) \subseteq \mathrm{WF}\left(u^{+}\right)$. Furthermore, $A_{x}^{\sigma}$, acting only in the $x$-variable, commutes with restriction to $t>0$, hence in intermediate steps we may consider $A_{x}^{\sigma}$ as convolution on $\mathbb{R}^{2}$ with $\mathcal{F}_{\xi \rightarrow x}^{-1}\left(b_{\beta}|\xi|^{\sigma}\right) \otimes \delta(t)$ and restrict to $t>0$ afterwards.

Note that we have $\tilde{u}=B_{x}^{\sigma} A_{x}^{\sigma} \tilde{u}=A_{x}^{\sigma} B_{x}^{\sigma} \tilde{u}$, where $B_{x}^{\sigma}$ is $x$-convolution with the inverse Fourier transform of the locally integrable function $\xi \mapsto 1 /\left(b_{\beta}|\xi|^{\sigma}\right)$. Thus, the statements (I) and (II) are equivalent to showing $\operatorname{WF}\left(G_{\sigma} u^{+}\right) \subseteq \mathrm{WF}\left(u^{+}\right)$and $\operatorname{WF}\left(G_{-\sigma} A_{x}^{\sigma} \tilde{u}^{+}\right) \subseteq \mathrm{WF}\left(A_{x}^{\sigma} \tilde{u}^{+}\right)$with $G_{\gamma}$ and $g_{\gamma}$ specified as in Remark 2.1(ii) with $\gamma=\sigma$ or $\gamma=-\sigma$ (both in the range $-1<\gamma<1$ ); clearly, $G_{\gamma}$ also commutes with restriction to $t>0$ and $A_{x}^{\sigma}=b_{\beta} G_{\sigma}, B_{x}^{\sigma}=G_{-\sigma} / b_{\beta}$.

The operator $G_{\gamma}$ can be considered as convolution on $\mathbb{R}^{2}$ with the distribution $g_{\gamma}(x) \otimes$ $\delta(t)$, where $\widehat{g_{\gamma}}(\xi)=|\xi|^{\gamma}$. The one-dimensional homogeneous distribution $g_{\gamma}$ can be determined explicitly via [5, Example 7.1.17], showing directly that $\operatorname{sing} \operatorname{supp}\left(g_{\gamma}\right)=\{0\}$, and we easily deduce from [5, Theorem 8.1.8.] that $\mathrm{WF}\left(g_{\gamma}\right)=\{(0, \xi) \mid \xi \neq 0\}$. Hence [5, Theorem 8.2.9] gives

$$
\mathrm{WF}\left(g_{\gamma} \otimes \delta\right) \subseteq\{(0,0 ; \xi, \tau) \mid(\xi, \tau) \neq(0,0)\} \cup\{(x, 0 ; 0, \tau) \mid x \in \mathbb{R}, \tau \neq 0\}
$$


and, recalling from Remark 2.1(ii) that $G_{\gamma} w=\left(g_{\gamma} \otimes \delta\right) * w$ is defined in case of $w=u^{+}$ or $w=A_{x}^{\sigma} \tilde{u}^{+}$, we may prove by cut-off techniques the appropriate extension of [5, Equation $(8.2 .16)]$ to these cases and obtain

$$
\mathrm{WF}\left(G_{\gamma} w\right) \subseteq \mathrm{WF}(w) \cup \underbrace{\{(x, t ; 0, \tau) \mid \exists y \in \mathbb{R}:(y, t ; 0, \tau) \in \mathrm{WF}(w)\}}_{=: \mathrm{WF}_{\text {vert }}(w)} .
$$

Equations (15) and (13) show $\mathrm{WF}_{\text {vert }}\left(\tilde{u}^{+}\right)=\emptyset$ and $\mathrm{WF}_{\text {vert }}\left(u^{+}\right)=\emptyset$, respectively, hence the proof of (I) and (II) is complete.

We may now put (I) and (II) to use with the outermost equalities in (16) and arrive at the following:

$$
\mathrm{WF}\left(\tilde{u}^{+}\right) \subseteq \mathrm{WF}\left(A_{x}^{\sigma} \tilde{u}^{+}\right)=\mathrm{WF}\left(\bar{Y} u^{+}\right) \subseteq \mathrm{WF}\left(u^{+}\right) .
$$

In summary, combining Equations (15) and (17) with (13) we obtain

$$
\begin{aligned}
\left\{(x, t ; \xi, 0) \mid t>0,(x, \xi) \in \mathrm{WF}\left(u_{0}\right)\right\}=\mathrm{WF}\left(\tilde{u}^{+}\right) \subseteq \mathrm{WF}\left(u^{+}\right) & \\
& \subseteq\left\{(x, t ; \xi, 0) \mid t>0,(x, \xi) \in \mathrm{WF}\left(u_{0}\right)\right\},
\end{aligned}
$$

hence we have, in fact, equality in all places of the above chain of inclusions, thereby the proof of the theorem in case $v_{0}=0$ is completed.

As shown in Lemma 2.2 the microlocal structure of $E_{1}^{+}$is equivalent to that of $E_{0}^{+}$, hence we have the same kind of wave front set statement with $v_{0}$ in place of $u_{0}$, if $u_{0}$ is smooth, since in this case $u^{+}=E_{1}^{+} *_{x} v_{0}$ plus a smooth contribution steming from $u_{0}$.

Finally, the solution in the general case $u_{0}, v_{0} \in \mathcal{E}^{\prime}(\mathbb{R})$ is just the sum of the two solutions for the special cases $v_{0}=0$ and $u_{0}=0$, hence its wave front set is contained in the corresponding union. Invariance of the wave front set under positive time translations follows in this case as well, since it was established via the operator factorization $Z=\bar{Y} \cdot Y$ with subsequent "symbol correction factor" $B$ and is valid for solutions $w$ of $Y B w=0$ independent of initial values.

Remark 2.6. The result on the wave front set of $u^{+}$in the above theorem implies, in particular, smoothness of $u^{+}$considered as a map from time into distributions on space (cf. [2, (23.65.5)]), i.e., $u^{+} \in C^{\infty}(] 0, \infty\left[, \mathcal{D}^{\prime}(\mathbb{R})\right)$; in addition, we have $u^{+}(t) \in \mathcal{S}^{\prime}(\mathbb{R})$ for every $t>0$.

\section{The time-fractional Zener wave equation}

For the special case of (11) when $\beta=1$ and $0 \leq \alpha<1$ we obtain the so-called time-fractional Zener wave equation

$$
Z u(x, t)=\partial_{t}^{2} u(x, t)-L_{t}^{\alpha} \partial_{x}^{2} u(x, t)=u_{0}(x) \otimes \delta^{\prime}(t)+v_{0}(x) \otimes \delta(t),
$$

whose unique solvability by distributions supported in a forward cone has been established in 9. Here we show a kind of non-characteristic regularity of the solution $u$ to problem (18).

The "Fourier symbol" of $Z$ is $z(\xi, \tau)=-\tau^{2}+l_{\alpha}(\tau) \xi^{2}$ with

$$
l_{\alpha}(\tau):=\frac{1+b e^{i \frac{\alpha \pi}{2}}(\tau-i 0)^{\alpha}}{1+a e^{i \frac{\alpha \pi}{2}}(\tau-i 0)^{\alpha}}=\frac{1+b i^{\alpha} \operatorname{sgn}(\tau)|\tau|^{\alpha}}{1+a i^{\alpha} \operatorname{sgn}(\tau)|\tau|^{\alpha}},
$$

to which we apply a conic cut-off to obtain a smooth symbol in both variables $(\xi, \tau)$, similarly as in Lemma 2.5 above. 
Lemma 3.1. Let $\Gamma \subseteq \mathbb{R}^{2}$ (representing the $(\xi, \tau)$-plane) be the union of a closed disc around $(0,0)$ and a closed narrow cone containg the $\xi$-axis and being symmetric with respect to both axes. Let $\Gamma^{\prime}$ be a closed set of the same shape as $\Gamma$, but with slightly larger disc and opening angle of the cone. Let $\tilde{b} \in S^{0}\left(\mathbb{R}^{2} \times \mathbb{R}^{2}\right)$ such that $\tilde{b}(x, t, \xi, \tau)$ is real, constant with respect to $(x, t)$, homogenous of degree 0 with respect to $(\xi, \tau)$ away from the disc contained in $\Gamma^{\prime}$, and such that $\tilde{b}(x, t, \xi, \tau)=0$, if $(\xi, \tau) \in \Gamma, \tilde{b}(x, t, \xi, \tau)=1$, if $(\xi, \tau) \notin \Gamma^{\prime}$. Then $p:=\tilde{b} z$ is a symbol belonging to the class $S^{2}\left(\mathbb{R}^{2} \times \mathbb{R}^{2}\right)$.

The proof is a variation of that of Lemma 2.5 .

Theorem 3.2. For the wave front set of $u^{+}$, the restriction of the solution $u$ to (18) to forward time $t>0$, we have the inclusion

$$
\mathrm{WF}\left(u^{+}\right) \subseteq\left\{(x, t ; \xi, \tau) \mid x \in \mathbb{R}, t>0, \xi \neq 0, \tau^{2}=\frac{b}{a} \xi^{2} \text { or } \tau=0\right\} .
$$

Proof. Let $B$ and $P$ be the pseudo-differential operators associated with the symbols $\tilde{b}$ and $p$, respectively, constructed in Lemma 3.1 according to arbitrary, but fixed, $\Gamma$ and $\Gamma^{\prime}$ chosen as specified there. We have $P=B Z$ and therefore

$$
P u^{+}=B Z u^{+}=0 .
$$

By non-characteristic regularity [8, Theorem 18.1.28],

$$
\mathrm{WF}(u) \subseteq \operatorname{Char}(P),
$$

where the characteristic set is $\operatorname{Char}(P)=\left(\mathbb{R}^{2} \times\left(\mathbb{R}^{2} \backslash\{(0,0)\}\right)\right) \backslash M$ with $M$ being defined as the set of all points $\left(x_{0}, t_{0}, \xi_{0}, \tau_{0}\right)$ such that there exist $c>0, R>0$ and a conic neighborhood $V$ of $\left(\xi_{0}, \tau_{0}\right)$ such that the following estimate holds:

$$
\forall(\xi, \tau) \in V, \xi^{2}+\tau^{2} \geq R^{2}: \quad|p(\xi, \tau)| \geq c\left(\xi^{2}+\tau^{2}\right) .
$$

1. We have $\mathbb{R}^{2} \times(\Gamma \backslash\{(0,0)\}) \cap M=\emptyset$, since $\tilde{b}\left(\xi_{0}, \tau_{0}\right)=0$ whenever $\left(\xi_{0}, \tau_{0}\right) \in \Gamma$. As $\Gamma$ gets more and more narrow (and smaller around the origin) only points of the form $\left(x_{0}, t_{0}, \xi_{0}, 0\right)$ will remain with this property.

2. We have no definite decay properties of the symbol in all of $\mathbb{R}^{2} \times\left(\Gamma^{\prime} \backslash \Gamma\right)$, but this will not be required as we let later shrink both $\Gamma^{\prime} \supset \Gamma$ to $\mathbb{R} \times\{0\}$, causing $\Gamma^{\prime} \backslash \Gamma \rightarrow \emptyset$.

3. Suppose $\left(x_{0}, t_{0}, \xi_{0}, \tau_{0}\right) \in \mathbb{R}^{2} \times\left(\mathbb{R}^{2} \backslash \Gamma^{\prime}\right)$, which will leave only points with $\tau_{0} \neq 0$ upon the shrinking process of $\Gamma^{\prime}$ and $\Gamma$.

(a) If $\tau_{0}^{2}=\frac{b}{a} \xi_{0}^{2}$, then the estimate (19) must fail in any conic neighborhood of $\left(\xi_{0}, \tau_{0}\right)$, since for $\lambda>0$ we have

$$
\left|p\left(\lambda \xi_{0}, \lambda \tau_{0}\right)\right|=\left|z\left(\lambda \xi_{0}, \lambda \tau_{0}\right)\right|=\lambda^{2} \xi_{0}^{2} \underbrace{\left|-\frac{b}{a}+\frac{b i^{\alpha} \operatorname{sgn}\left(\tau_{0}\right)\left|\tau_{0}\right|^{\alpha}+\lambda^{-\alpha}}{a i^{\alpha} \operatorname{sgn}\left(\tau_{0}\right)\left|\tau_{0}\right|^{\alpha}+\lambda^{-\alpha}}\right|}_{=: d(\lambda)},
$$

where $d(\lambda) \rightarrow 0$ as $\lambda \rightarrow \infty$, which makes a lower bound of the form $\left|p\left(\lambda \xi_{0}, \lambda \tau_{0}\right)\right| \geq c \lambda^{2}$ to hold for all $\lambda \geq R / \sqrt{\xi_{0}^{2}+\tau_{0}^{2}}$ impossible. Thus, $\left(x_{0}, t_{0}, \xi_{0}, \tau_{0}\right) \notin M$. 
(b) If $\tau_{0}^{2} \neq \frac{b}{a} \xi_{0}^{2}$, we define a closed conic neighborhood of the point $\left(\xi_{0}, \tau_{0}\right)$ by $V:=$ $\left\{(\xi, \tau) \in \mathbb{R}^{2}|| \frac{\tau^{2}-\frac{b}{a} \xi^{2}}{\xi^{2}+\tau^{2}} \mid \geq c_{0}-\delta\right\}$, where $c_{0}:=\left|\frac{\tau_{0}^{2}-\frac{b}{a} \xi_{0}^{2}}{\xi_{0}^{2}+\tau_{0}^{2}}\right|$ and $0<\delta<c_{0}$. Let $V_{R}:=$ $V \cap\left\{(\xi, \tau) \mid \xi^{2}+\tau^{2} \geq R^{2}\right\}$ and suppose that $R>0$ is large enough and $\delta$ chosen sufficiently small to ensure $V_{R} \cap \Gamma^{\prime}=\emptyset$ as well as $V_{R} \cap\left\{(\xi, \tau) \mid \tau^{2}=\frac{b}{a} \xi^{2}\right\}=\emptyset$.

Let $(\xi, \tau) \in V_{R}$, then $\tau^{2} \geq\left(c_{0}-\delta\right)\left(\xi^{2}-\tau^{2}\right)+\frac{b}{a} \xi^{2} \geq \min \left(c_{0}-\delta, \frac{b}{a}\right)\left(\xi^{2}+\tau^{2}\right) \geq c_{1} R^{2}$. Since $l_{\alpha}(\tau) \rightarrow \frac{b}{a}(|\tau| \rightarrow \infty)$ we may thus choose $R$ large enough to have $l_{\alpha}(\tau)-\frac{b}{a}<\frac{c_{0}-\delta}{2}$, if $\xi^{2}+\tau^{2} \geq R^{2}$ and $(\xi, \tau) \in V$. Thus, $(\xi, \tau) \in V_{R}$ implies

$$
\begin{aligned}
& |p(\xi, \tau)|=|z(\xi, \tau)|=\left|\tau^{2}-l_{\alpha}(\tau) \xi^{2}\right|=\left|\tau^{2}-\frac{b}{a} \xi^{2}-\left(l_{\alpha}(\tau)+\frac{b}{a}\right) \xi^{2}\right| \\
& \geq\left|\tau^{2}-\frac{b}{a} \xi^{2}\right|-\left|l_{\alpha}(\tau)+\frac{b}{a}\right| \xi^{2} \geq\left(c_{0}-\delta\right)\left(\xi^{2}+\tau^{2}\right)-\frac{c_{0}-\delta}{2} \xi^{2} \geq \frac{c_{0}-\delta}{2}\left(\xi^{2}+\tau^{2}\right) .
\end{aligned}
$$

Therefore we have in this case $\left(x_{0}, t_{0}, \xi_{0}, \tau_{0}\right) \in M$.

To summarize,

$$
\mathrm{WF}\left(u^{+}\right) \subseteq \operatorname{Char}(P) \subseteq \mathbb{R}^{2} \times\left((\Gamma \backslash\{(0,0)\}) \cup\left(\Gamma^{\prime} \backslash \Gamma\right) \cup\left\{\left(\xi_{0}, \tau_{0}\right) \mid \tau_{0}^{2}=\frac{b}{a} \xi_{0}^{2}\right\}\right) .
$$

This result holds for any $\Gamma$ and $\Gamma^{\prime}$ chosen arbitrarily according to the specifications in the previous lemma. Letting $\Gamma^{\prime} \supseteq \Gamma$ both shrink toward the $\xi$-axis yields the claim of the theorem, since we may use the intersection of all corresponding $\left(\Gamma, \Gamma^{\prime}\right)$-dependent sets in the middle and in the right part of the above chain of inclusions.

\section{Acknowledgement}

This research is supported by project P25326 of the Austrian Science Fund and by projects 174005,174024 of the Serbian Ministry of Education and Science, as well as by project 114 $451-947$ of the Secretariat for Science of Vojvodina.

\section{References}

[1] T. M. Atanackovic, M. Janev, Lj. Oparnica, S. Pilipovic, and D. Zorica. Space-time fractional Zener wave equation. Proceedings of the Royal Society A: Mathematical, Physical and Engineering Sciences, 471:20140614-1-25, 2015.

[2] J. Dieudonné. Treatise on Analysis, volume VIII. Academic Press, Boston, 1993.

[3] J. Duoandikoetxea. Fourier Analysis. American Mathematical Society, Providence, 2001.

[4] L. Hörmander. Fourier integral operators. I. Acta Mathematica, 127:79-183, 1971.

[5] L. Hörmander. The Analysis of Linear Partial Differential Operators I. Distribution Theory and Fourier Analysis. Springer-Verlag, Berlin, 1983.

[6] L. Hörmander. The Analysis of Linear Partial Differential Operators II. Differential Operators with Constant Coefficients. Springer-Verlag, Berlin, 1983.

[7] L. Hörmander. The Analysis of Linear Partial Differential Operators IV. Fourier Integral Operators. Springer-Verlag, Berlin, 1985. 
[8] L. Hörmander. The Analysis of Linear Partial Differential Operators III. Pseudo-Differential Operators. Springer-Verlag, Berlin, 1994.

[9] S. Konjik, Lj. Oparnica, and D. Zorica. Waves in fractional Zener type viscoelastic media. Journal of Mathematical Analysis and Applications, 365:259-268, 2010.

[10] E. M. Stein. Singular Integrals and Differential Properties of Functions. Princeton University Press, Princeton, New Jersey, 1970. 